\title{
Analisi di budget impact del biosimilare di adalimumab: lo scenario italiano
}

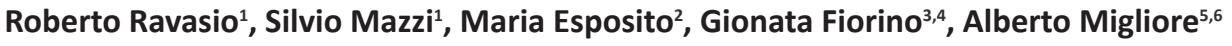 \\ ${ }^{1}$ Health Publishing \& Services Srl, Milano - Italy \\ ${ }^{2}$ UOSD Dermatologia Generale ed Oncologica, Università degli Studi dell'Aquila, L'Aquila - Italy \\ ${ }^{3}$ IBD Center, Dipartimento di Gastroenterologia, Humanitas Research Hospital, Rozzano (MI) - Italy \\ ${ }^{4}$ Department of Biomedical Sciences, Humanitas University, Rozzano (MI) - Italy \\ ${ }_{5}^{5}$ Responsabile UO di Reumatologia, Ospedale San Pietro FBF, Roma - Italy \\ ${ }^{6}$ Presidente ISPOR Italy-Rome Chapter, Roma - Italy
}

\begin{abstract}
Introduction: Patients' quality of life impairment together with a heavy burden to the NHS and society are caused by chronic inflammatory diseases. Their treatment substantially improved with the advent of biological drugs (such as adalimumab), but these drugs are very expensive. At present, the patent protection of adalimumab (Humira ${ }^{\circledR}$ ) has expired, and a biosimilar $\left(\right.$ Hyrimoz $\left.^{\circledR}\right)$ has been approved. Since the biosimilar price is expected to be lower as compared with the originator's, the present Drug Budget Impact analysis tries to evaluate whether and how much profitable the biosimilar availability will be for the Italian NHS, in terms of cost containment (savings).

Methods and results: The time horizon in the analysis model is five years. The initial overall number of patients treated with adalimumab is estimated (based on the Italian population, the disease prevalence rates, the proportion of patients under treatment and adalimumab market share) and kept constant in time. However, the model assumes that, year by year, patients in treatment with the originator will partly switch to the biosimilar (based on a prudent estimate of the uptake rate), while (due to a bolder estimate) a larger share of naïve patients will directly start with the biosimilar.

Thanks to such assumptions, total number of patients treated with adalimumab (separately with the originator and with the biosimilar) is estimated, with the respective total costs.

Conclusions: According to the present analysis, the availability of the biosimilar would generate cumulated savings (in five years) as high as $€ 260$ millions for the Italian NHS.
\end{abstract}

Keywords: Adalimumab, Anti-TNF, Biosimilar, Cost, Italian NHS

\section{Introduzione e obiettivi}

Le malattie infiammatorie autoimmuni croniche determinano disabilità elevata sin dalle fasi iniziali, con sensibili ripercussioni sulla qualità di vita non solo degli stessi pazienti, ma anche dei famigliari e, più in generale, delle persone che se ne occupano $(1,2)$. Artrite reumatoide (AR), spondilite anchilosante (SA), artrite psoriasica (PSA), psoriasi (PSO), malattia di Crohn (MC) e colite ulcerosa (CU) rientrano in questo gruppo di patologie.

Poiché croniche, sono malattie a forte impatto sia per il Sistema Sanitario che per la Società. Si stima che la parte prevalente dei costi sia costituita dalle assenze dal posto di

Received: March 27, 2019

Accepted: April 10, 2019

Published online: May 6, 2019

Corresponding author

Roberto Ravasio

Health Publishing \& Services Srl

Piazza Duca d'Aosta 12

20124 Milano, Italy

rravasio@aboutpharma.com lavoro e dalle cure informali (3), mentre i costi diretti sanitari rappresentino meno della metà del totale (4-6).

Pur essendo incidenza e prevalenza di queste malattie non particolarmente elevate, esse corrispondono nel panorama nazionale a una frazione non secondaria della popolazione. Per alcune di esse si riscontrano prevalenze più elevate nel Nord Europa (7).

L'attuale terapia farmacologica permette di raggiungere lo stato di remissione o la bassa attività di malattia, con blocco della progressione del danno e prevenzione delle complicanze e comorbilità associate alle patologie. L'arrivo alla fine degli anni ' 90 dei farmaci biologici, in particolare degli inibitori del Tumor Necrosis Factor- $\alpha$ (anti TNF- $\alpha$ ), ha sensibilmente cambiato la storia del trattamento di queste patologie. Questi farmaci, fin da subito dimostratisi estremamente efficaci e con un buon profilo di sicurezza, costituiscono però una delle voci di costo più rilevanti a carico del Servizio Sanitario Nazionale (SSN). Infatti, anche per gli elevati costi, i farmaci biologici trovano spesso indicazione solo dopo il fallimento della terapia convenzionale (es. metotrexato per l'AR; mesalazina e/o azatioprina per le malattie infiammatorie intestinali; ciclosporina, metotrexato per PSA e PSO oltre alla fototerapia con UVB o PUVA esclusiva- 
mente per la forma cutanea di psoriasi) o nei soggetti per i quali vi sono controindicazioni all'uso della terapia convenzionale.

In tema di sostenibilità quindi, la scadenza della protezione brevettuale di recenti farmaci biologici ha spinto le aziende farmaceutiche a investire risorse nella produzione del corrispettivo farmaco "biosimilare". L'European Medicines Agency (EMA) ha provveduto a emanare linee guida (Guideline on Similar Biological Medicinal Products) (8-11) specificando l'iter a cui deve essere sottoposto un farmaco biosimilare per ottenere l'autorizzazione all'immissione in commercio: l'esercizio di comparabilità. Questo esercizio è basato su un solido confronto diretto tra il biosimilare e il medicinale originatore di riferimento in termini di qualità, sicurezza ed efficacia clinica e non clinica. La comparabilità della qualità è determinata rispetto alla struttura molecolare oltre che rispetto alla funzionalità, e deve essere dimostrata con un'esauriente caratterizzazione analitica e con studi e test biologici sui legami dei recettori coinvolti, da eseguirsi in modo strettamente comparativo sul biosimilare e sul medicinale di riferimento. La comparabilità clinica e non clinica garantiscono che eventuali differenze osservate in termini di qualità non abbiano alcun impatto sulla sicurezza ed efficacia del medicinale biosimilare rispetto al medicinale originatore di riferimento.

Adalimumab, farmaco biologico antinfiammatorio anti TNF- $\alpha$, è indicato per il trattamento di tutte le malattie infiammatorie croniche considerate nel presente studio. Adalimumab è il terzo, dopo infliximab ed etanercept, dei farmaci biologici anti TNF- $\alpha$ e il primo anticorpo monoclonale interamente umano approvato dalla Food and Drug Administration (FDA) per il trattamento dell'artrite reumatoide (2002).

In Europa, alcuni biosimilari di adalimumab sono disponibili dalla seconda metà del 2018, mentre negli USA si dovrà attendere fino al 2023. Dal momento che i farmaci biosimilari sono comparabili in termini di efficacia e sicurezza rispetto all'originatore, il beneficio atteso dalla loro immissione in commercio è rappresentato principalmente dal loro minore prezzo, e quindi dal conseguente effetto competitivo sulla spesa farmaceutica pubblica.

Un aggiornamento sulla penetrazione dei biosimilari è offerto da un recente rapporto del Centro Studi IQVIA Italia, nel quale si evidenzia come, soprattutto nell'ultimo decennio, il crescente utilizzo dei farmaci biosimilari abbia ricoperto un importante ruolo tra innovazione e sostenibilità della spesa farmaceutica (12). Nel mercato europeo EU5 (Gran Bretagna, Francia, Italia, Germania, Spagna), I'Italia si colloca in una posizione medio-alta nella classifica relativa alla penetrazione dei biosimilari (12). Ad esempio, con riferimento a infliximab, lanciato nel 2015, I'Italia ha raggiunto nel 2017 una penetrazione del 61\% - seconda a Gran Bretagna (88\%), ma prima di Germania, Spagna e Francia, dove la penetrazione è inferiore al 49\% (12). Ipotizzando una riduzione del prezzo del $20 \%$ dettata dalla competizione diretta dei biosimilari per gli 8 biologici che hanno perso o perderanno la protezione brevettuale tra il 2017 e il 2022 (adalimumab, trastuzumab, bevacizumab, enoxaparina sodica, insulina lispro, ranibizumab, teriparatide e pegfilgrastim), il risparmio medio generato sarebbe pari a quasi 60 milioni di euro per anno, nei prossimi 5 anni (che salirebbe a 90 milioni se tale riduzione fosse del 30\%) (12).

Secondo un'altra fonte (13), nei primi 9 mesi del 2018 le 10 molecole biosimilari in commercio sul mercato italiano (epoetine, filgrastim, somatropina, follitropina alfa, infliximab, insulina glargine, etanercept, rituximab, enoxaparina e insulina lispro), per un totale di 45 prodotti, hanno assorbito il $14 \%$ dei consumi nazionali contro l' $86 \%$ detenuto dai corrispondenti originatori. Se dalla quota di mercato si passa al trend - e considerando i biosimilari al netto delle new entry nel corso del 2017 di enoxaparina e insulina lispro - i consumi di biosimilari risultano cresciuti del 51\% rispetto ai primi 9 mesi del 2017, a fronte di una contrazione dei biologici originatori del $9 \%$ (13).

Da ciò consegue l'obiettivo del presente studio: valutare quale contenimento della spesa stessa potrebbe derivare dai potenziali risparmi - Drug Budget Impact (DBI) - generati in Italia nei prossimi 5 anni dall'utilizzo del farmaco adalimumab biosimilare (di seguito "biosimilare"). La sovrapponibilità terapeutica rende non rilevanti le valutazioni sui costi di eventi evitati ed effetti collaterali.

\section{Materiali e metodi}

Per stimare l'impatto (DBI) del biosimilare di adalimumab (Hyrimoz ${ }^{\circledR}$ ) è stato costruito un modello, nel quale il farmaco biosimilare, meno costoso dell'originatore, possa in parte sostituirsi al biologico di riferimento (Humira ${ }^{\circledR}$ ). Per effetto di questa sostituzione si genererebbero dei risparmi (solo costo di trattamento) per il SSN, quantificabili confrontando la spesa a suo carico nelle ipotesi, rispettivamente, di assenza e di presenza sul mercato del biosimilare. Nella prima ipotesi la spesa è costituita dal solo costo dei pazienti trattati con l'originatore. Nella seconda ipotesi tale spesa è invece composta dal costo dei pazienti trattati col biosimilare in aggiunta a quello dei rimanenti pazienti trattati con l'originatore.

L'orizzonte temporale del DBI copre un periodo di 5 anni a partire dal primo anno di commercializzazione del biosimilare di adalimumab in relazione alle seguenti sei indicazioni terapeutiche: artrite reumatoide, spondilite anchilosante, artrite psoriasica, psoriasi, malattia di Crohn e colite ulcerosa.

La popolazione oggetto del presente DBI è stata stimata distinguendo tra due tipologie di pazienti: 1) quella dei pazienti che iniziano la prima linea di trattamento biologico con adalimumab (di seguito naïve population), per i quali l'adozione del biosimilare potrà avvenire fin da subito al posto dell'originatore e 2) quella dei pazienti già in trattamento con un biologico (di seguito switch population). Quest'ultima tipologia di pazienti è stata distinta in due ulteriori sottogruppi: a) pazienti in trattamento di prima linea con l'originatore di adalimumab (di seguito pazienti in trattamento continuativo), per i quali il passaggio al biosimilare può avvenire tramite switch e b) pazienti che, fallita una prima linea biologica, effettuano lo switch ad adalimumab (di seguito pazienti switch win). Per questi ultimi l'adozione del biosimilare potrà avvenire fin da subito al posto dell'originatore. Con l'obiettivo di riprodurre uno scenario il più realistico possibile, la scelta di distinguere tra differenti popolazioni e sottopopolazioni ha permesso ai tre clinici (1 dermatologo, 1 gastroenterologo e 1 reumatologo), coinvolti nello studio in qualità di esperti, di stimare dei tassi di uptake per i pazienti in trattamento continuativo più prudenti rispetto a quelli adottati per la naïve population e per i pazienti switch win. 
La stima del numero di pazienti oggetto dell'analisi di budget impact è stata effettuata partendo dalla popolazione residente in Italia al $1^{\circ}$ gennaio 2018 (base campionaria); tale popolazione viene mantenuta costante negli anni successivi. A partire dal primo anno, la switch population (nelle sue due componenti: trattamento continuativo e switch win) e la naïve population sono state dimensionate in funzione dei relativi tassi di prevalenza dei pazienti trattati con biologico e - tra questi - di quelli trattati con adalimumab originatore. Applicando a queste popolazioni i rispettivi tassi annuali di uptake ipotizzati, è stato infine ottenuto il numero di soggetti potenzialmente trattabili con il biosimilare di adalimumab e, per differenza, quelli mantenuti con l'originatore. Partendo dalla popolazione italiana, la Tabella I riporta, per ciascuna malattia, i tassi epidemiologici utilizzati per stimare i pazienti oggetto di analisi. Come per i tassi di uptake, anche i tassi epidemiologici riflettono la stima del panel di esperti. I tassi di uptake ipotizzati nel modello sono invece riportati nella Tabella II.

Il costo medio di trattamento per paziente/anno sia dell'originatore sia del biosimilare di adalimumab riflette il prezzo ex-factory al netto degli sconti obbligatori di legge e il numero di dosi somministrate in un anno. Specificamente, il costo di 1 penna / 1 siringa da $40 \mathrm{mg}$ di adalimumab originatore è pari a $€ 482,19$, mentre quello del biosimilare a $€ 342,35$. La Tabella III riporta, suddividendo per patologia, le quantità di farmaco necessarie per il trattamento annuo di un paziente.

La Figura 1 illustra schematicamente la struttura del DBI. L'analisi è stata condotta osservando quanto indicato dalle linee guida dell'International Society for Pharmacoeconomics and Outcomes Research (ISPOR) per la conduzione di Budget Impact Analysis (BIA) (14,15).

\section{Risultati}

L'analisi di DBI del biosimilare è stata fatta sia a livello complessivo (aggregazione delle sei indicazioni terapeutiche) (Tab. IV) che di singole indicazioni terapeutiche (Tab. da V a X).

Sulla base dei tassi epidemiologici di Tabella I, il numero complessivo dei pazienti in trattamento con adalimumab nel primo anno di osservazione del DBI risulta di 23.250, di cui

TABELLA I - Tassi epidemiologici - Pazienti trattati con biologici

\begin{tabular}{|c|c|c|c|c|c|c|}
\hline Indicazione terapeutica & AR & SA & PSA & PSO & MC & CU \\
\hline Pazienti in trattamento con biologico (continuativo) (\%) & 0,0421 & 0,0111 & 0,0302 & 0,2520 & 0,0074 & 0,0040 \\
\hline - di cui in trattamento con adalimumab originatore (\%) & 32,70 & 32,92 & 17,53 & 0,28 & 23,48 & 5,90 \\
\hline Pazienti in trattamento con biologico (switch win) (\%) & 0,0063 & 0,0036 & 0,0012 & 0,1512 & 0,0061 & 0,0020 \\
\hline - di cui in switch win con adalimumab originatore (\%) & 15,93 & 35,45 & 60,80 & 0,25 & 32,38 & 6,50 \\
\hline Pazienti naïve ai biologici (\%) & 0,0082 & 0,0035 & 0,004 & 0,1008 & 0,0112 & 0,014 \\
\hline - di cui in trattamento con adalimumab originatore (\%) & 20,00 & 30,00 & 20,00 & 0,02 & 31,50 & 3,00 \\
\hline
\end{tabular}

$\mathrm{AR}$ = artrite reumatoide; $\mathrm{CU}$ = colite ulcerosa; $\mathrm{MC}$ = malattia di Crohn; $\mathrm{PSA}=$ artrite psoriasica; $\mathrm{PSO}$ = psoriasi; $\mathrm{SA}=$ spondilite anchilosante .

TABELLA II - Tassi di uptake del biosimilare vs l'originatore

\begin{tabular}{|c|c|c|c|c|c|}
\hline Popolazione & I anno & II anno & III anno & IV anno & V anno \\
\hline \multicolumn{6}{|l|}{ Artrite reumatoide } \\
\hline Pazienti in trattamento con adalimumab (continuativo) & $20 \%$ & $30 \%$ & $40 \%$ & $65 \%$ & $80 \%$ \\
\hline Pazienti naïve al biologico che vengono trattati con adalimumab (naïve population) & $70 \%$ & $80 \%$ & $90 \%$ & $100 \%$ & $100 \%$ \\
\hline \multicolumn{6}{|l|}{ Spondilite anchilosante } \\
\hline Pazienti in trattamento con biologico diverso da adalimumab (switch win) & $70 \%$ & $80 \%$ & $90 \%$ & $100 \%$ & $100 \%$ \\
\hline Pazienti naïve al biologico che vengono trattati con adalimumab (naïve population) & $70 \%$ & $80 \%$ & $90 \%$ & $100 \%$ & $100 \%$ \\
\hline \multicolumn{6}{|l|}{ Artrite psoriasica } \\
\hline Pazienti in trattamento con adalimumab (continuativo) & $20 \%$ & $30 \%$ & $40 \%$ & $65 \%$ & $80 \%$ \\
\hline Pazienti in trattamento con adalimumab (continuativo) & $25 \%$ & $35 \%$ & $45 \%$ & $70 \%$ & $85 \%$ \\
\hline Pazienti in trattamento con biologico diverso da adalimumab (switch win) & $70 \%$ & $80 \%$ & $90 \%$ & $100 \%$ & $100 \%$ \\
\hline Pazienti naïve al biologico che vengono trattati con adalimumab (naïve population) & $70 \%$ & $80 \%$ & $90 \%$ & $100 \%$ & $100 \%$ \\
\hline
\end{tabular}


TABELLA II - (Continua)

\begin{tabular}{|c|c|c|c|c|c|}
\hline Popolazione & I anno & II anno & III anno & IV anno & V anno \\
\hline \multicolumn{6}{|l|}{ Malattia di Crohn } \\
\hline Pazienti in trattamento con adalimumab (continuativo) & $30 \%$ & $40 \%$ & $50 \%$ & $75 \%$ & $90 \%$ \\
\hline Pazienti in trattamento con biologico diverso da adalimumab (switch win) & $70 \%$ & $80 \%$ & $90 \%$ & $100 \%$ & $100 \%$ \\
\hline Pazienti naïve al biologico che vengono trattati con adalimumab (naïve population) & $70 \%$ & $80 \%$ & $90 \%$ & $100 \%$ & $100 \%$ \\
\hline \multicolumn{6}{|l|}{ Colite ulcerosa } \\
\hline Pazienti in trattamento con adalimumab (continuativo) & $30 \%$ & $40 \%$ & $50 \%$ & $75 \%$ & $90 \%$ \\
\hline Pazienti in trattamento con biologico diverso da adalimumab (switch win) & $70 \%$ & $80 \%$ & $90 \%$ & $100 \%$ & $100 \%$ \\
\hline Pazienti naïve al biologico che vengono trattati con adalimumab (naïve population) & $70 \%$ & $80 \%$ & $90 \%$ & $100 \%$ & $100 \%$ \\
\hline
\end{tabular}

TABELLA III - Dosaggio annuale per indicazione terapeutica

\begin{tabular}{lccc}
\hline Indicazione terapeutica & $\begin{array}{c}\text { Dose per } \\
\text { somm. } \\
\text { (mg) }\end{array}$ & $\begin{array}{c}\text { Intervallo } \\
\text { di somm. } \\
\text { (giorni) }\end{array}$ & $\begin{array}{c}\text { N. di } \\
\text { somm. } \\
\text { annuali }\end{array}$ \\
\hline Artrite reumatoide & 40 & 14 & 26 \\
Spondilite anchilosante & 40 & 14 & 26 \\
Artrite psoriasica & 40 & 14 & 26 \\
Psoriasi & 40 & 14 & 26 \\
Malattia di Crohn & 40 & 14 & 26 \\
Colite ulcerosa & 40 & 14 & 26 \\
\hline
\end{tabular}

Somm. = somministrazione.
14.512 trattati con l'originatore e 8.739 con il biosimilare. Sempre nello stesso anno, i pazienti incidenti sarebbero 4.563, di cui 1.369 trattati con l'originatore e 3.194 con il biosimilare di adalimumab. Assumendo che nei restanti quattro anni di osservazione si realizzino i tassi di uptake ipotizzati in Tabella II, nel quinto e ultimo anno saranno complessivamente passati al biosimilare 20.320 pazienti (contro gli 8.739 del primo anno) (Tab. IV). I pazienti trattati con l'originatore diminuiranno invece nel corso del quinquennio da 14.512 a 2.930 (Tab. IV). In termini di DBI, per il SSN il costo complessivo per trattare nel primo anno i 23.250 pazienti, se il biosimilare non fosse disponibile, sarebbe pari a €292.285.991. Costo che si ridurrebbe invece $\mathrm{a} € 260.426 .933$, grazie al minore prezzo del biosimilare, qualora questo si sostituisse in parte

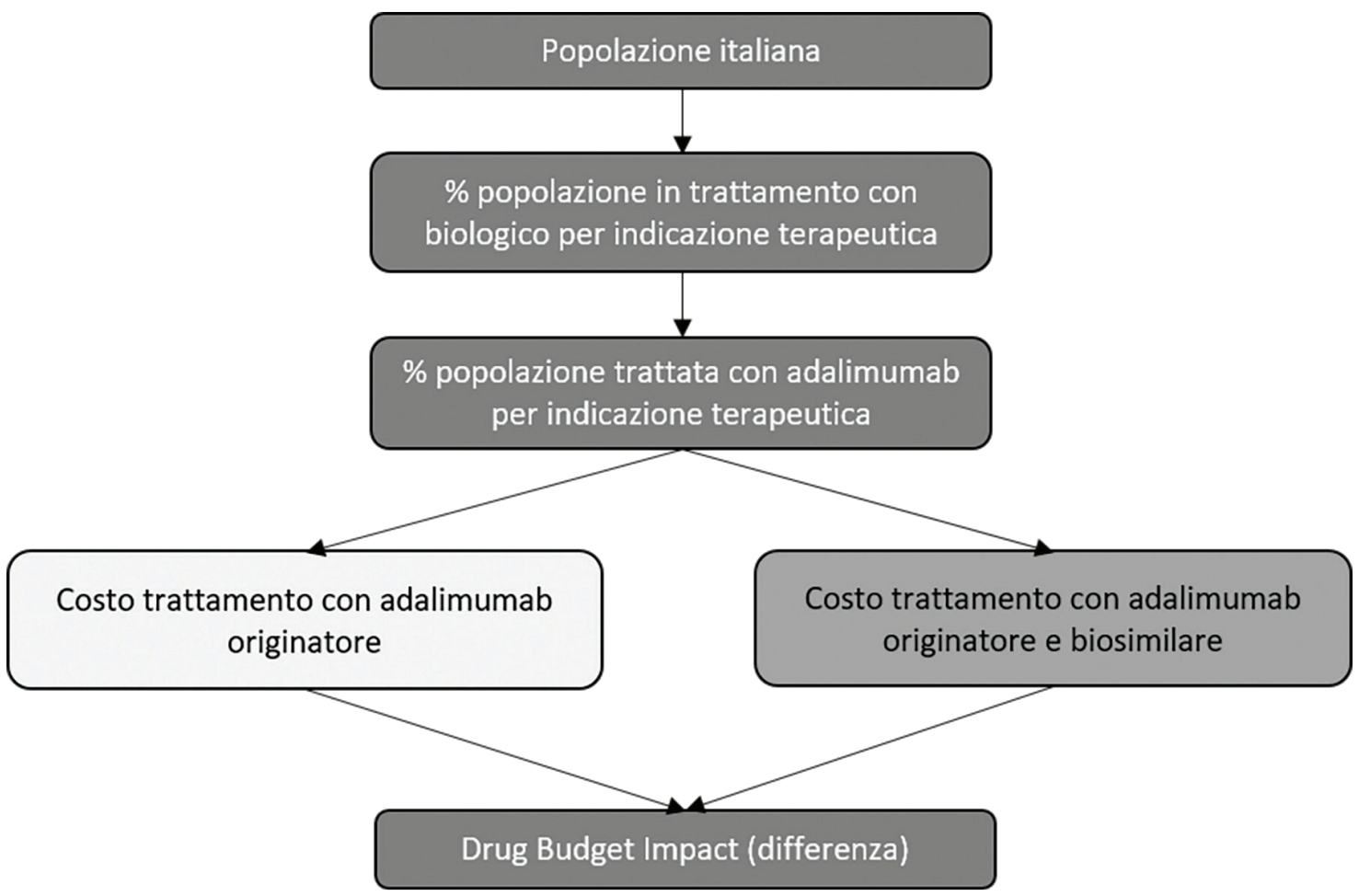

Fig. 1 - Struttura del modello di Drug Budget Impact. 
TABELLA IV - Drug Budget Impact: risultati complessivi

\begin{tabular}{|c|c|c|c|c|c|}
\hline Parametri & I anno & Il anno & III anno & IV anno & V anno \\
\hline Pazienti totali in trattamento con adalimumab & 23.250 & 23.250 & 23.250 & 23.250 & 23.250 \\
\hline Pazienti trattati con adalimumab biosimilare & 8.739 & 11.064 & 13.389 & 18.017 & 20.320 \\
\hline Pazienti trattati con adalimumab originatore & 14.512 & 12.187 & 9.862 & 5.233 & 2.930 \\
\hline cenario senza biosimilare (solo originatore) & $€ 292.285 .991$ & $€ 292.285 .991$ & $€ 292.285 .991$ & $€ 292.285 .991$ & $€ 292.285 .991$ \\
\hline Scenario con biosimilare (originatore + biosimilare) & $€ 260.426 .933$ & $€ 251.950 .396$ & $€ 243.473 .859$ & $€ 226.599 .921$ & $€ 218.202 .519$ \\
\hline - di cui costo pazienti trattati con biosimilare & $€ 77.996 .612$ & $€ 98.748 .674$ & $€ 119.500 .736$ & $€ 160.811 .122$ & $€ 181.369 .445$ \\
\hline - di cui costo pazienti trattati con originatore & $€ 182.430 .321$ & $€ 153.201 .721$ & $€ 123.973 .122$ & $€ 65.788 .799$ & $€ 36.833 .074$ \\
\hline Risparmio annuo & $€ 31.859 .059$ & $€ 40.335 .596$ & $€ 48.812 .133$ & $€ 65.686 .071$ & $€ 74.083 .472$ \\
\hline Risparmio cumulato & $€ 31.859 .059$ & $€ 72.194 .654$ & $€ 121.006 .787$ & $€ 186.692 .857$ & $€ 260.776 .329$ \\
\hline $\begin{array}{l}\text { Pazienti in trattamento con adalimumab } \\
\text { (continuativo + switch win) }\end{array}$ & 18.687 & 18.687 & 18.687 & 18.687 & 18.687 \\
\hline Pazienti trattati con adalimumab biosimilare & 5.544 & 7.413 & 9.282 & 13.454 & 15.757 \\
\hline Pazienti trattati con adalimumab originatore & 13.143 & 11.274 & 9.405 & 5.233 & 2.930 \\
\hline Scenario senza biosimilare (solo originatore) & $€ 234.922 .817$ & $€ 234.922 .817$ & $€ 234.922 .817$ & $€ 234.922 .817$ & $€ 234.922 .817$ \\
\hline Scenario con biosimilare (originatore + biosimilare) & $€ 214.708 .817$ & $€ 207.895 .859$ & $€ 201.082 .902$ & $€ 185.872 .544$ & $€ 177.475 .143$ \\
\hline - di cui costo pazienti trattati con biosimilare & $€ 49.487 .448$ & $€ 66.166 .773$ & $€ 82.846 .097$ & $€ 120.083 .745$ & $€ 140.642 .069$ \\
\hline - di cui costo pazienti trattati con originatore & $€ 165.221 .368$ & $€ 141.729 .087$ & $€ 118.236 .805$ & $€ 65.788 .799$ & $€ 36.833 .074$ \\
\hline Risparmio annuo & $€ 20.214 .000$ & $€ 27.026 .957$ & $€ 33.839 .914$ & $€ 49.050 .273$ & $€ 57.447 .674$ \\
\hline Risparmio cumulato & $€ 20.214 .000$ & $€ 47.240 .957$ & $€ 81.080 .872$ & $€ 130.131 .144$ & $€ 187.578 .818$ \\
\hline Pazienti naïve trattati con adalimumab & 4.563 & 4.563 & 4.563 & 4.563 & 4.563 \\
\hline Pazienti trattati con adalimumab biosimilare & 3.194 & 3.650 & 4.107 & 4.563 & 4.563 \\
\hline Pazienti trattati con adalimumab originatore & 1.369 & 913 & 456 & 0 & 0 \\
\hline Scenario senza biosimilare (solo originatore) & $€ 57.363 .175$ & $€ 57.363 .175$ & $€ 57.363 .175$ & $€ 57.363 .175$ & $€ 57.363 .175$ \\
\hline Scenario con biosimilare (originatore + biosimilare) & $€ 45.718 .116$ & $€ 44.054 .536$ & $€ 42.390 .956$ & $€ 40.727 .377$ & $€ 40.727 .377$ \\
\hline - di cui costo pazienti trattati con biosimilare & $€ 28.509 .164$ & $€ 32.581 .901$ & $€ 36.654 .639$ & $€ 40.727 .377$ & $€ 40.727 .377$ \\
\hline - di cui costo pazienti trattati con originatore & $€ 17.208 .952$ & $€ 11.472 .635$ & $€ 5.736 .317$ & $€ 0$ & $€ 0$ \\
\hline Risparmio annuo & $€ 11.645 .059$ & $€ 13.308 .638$ & $€ 14.972 .218$ & $€ 16.635 .798$ & $€ 16.635 .798$ \\
\hline Risparmio cumulato & $€ 11.645 .059$ & $€ 24.953 .697$ & $€ 39.925 .915$ & $€ 56.561 .713$ & $€ 73.197 .511$ \\
\hline
\end{tabular}

TABELLA V - Drug Budget Impact: risultati per l'artrite reumatoide

\begin{tabular}{lcc}
\hline Parametri & I anno & V anno \\
\hline Pazienti totali in trattamento con adalimumab & $\mathbf{9 . 9 2 0}$ & $\mathbf{9 . 9 2 0}$ \\
Pazienti trattati con adalimumab biosimilare & 2.785 & 8.256 \\
Pazienti trattati con adalimumab originatore & 7.135 & 1.664 \\
Scenario senza biosimilare (solo originatore) & $€ 124.706 .884$ & $€ 124.706 .884$ \\
Scenario con biosimilare (originatore + biosimilare) & $€ 114.554 .507$ & $€ 94.606 .389$ \\
- di cui costo pazienti trattati con biosimilare & $€ 24.854 .817$ & $€ 73.691 .339$ \\
- di cui costo pazienti trattati con originatore & $€ 89.699 .690$ & $€ 20.915 .050$ \\
Risparmio annuo & $€ 10.152 .378$ & $€ 30.100 .496$ \\
Risparmio cumulato & $€ 10.152 .378$ & $€ 96.958 .781$ \\
\hline
\end{tabular}


TABELLA VI - Drug Budget Impact: risultati per la spondilite anchilosante

\begin{tabular}{lcc}
\hline Parametri & I anno & V anno \\
\hline $\begin{array}{l}\text { Pazienti totali in trattamento con } \\
\text { adalimumab }\end{array}$ & $\mathbf{3 . 6 1 3}$ & $\mathbf{3 . 6 1 3}$ \\
$\begin{array}{l}\text { Pazienti trattati con adalimumab } \\
\text { biosimilare }\end{array}$ & 1.426 & 3.172 \\
$\begin{array}{l}\text { Pazienti trattati con adalimumab } \\
\text { originatore }\end{array}$ & 2.187 & 441 \\
$\begin{array}{l}\text { Scenario senza biosimilare } \\
\text { (solo originatore) }\end{array}$ & $€ 45.424 .786$ & $€ 45.424 .786$ \\
$\begin{array}{l}\text { Scenario con biosimilare } \\
\text { (originatore + biosimilare) }\end{array}$ & $€ 40.224 .558$ & $€ 33.859 .727$ \\
- di cui costo pazienti trattati con & & \\
$\quad$ biosimilare & $€ 12.731 .077$ & $€ 28.313 .309$ \\
- di cui costo pazienti trattaticon & & \\
$\quad$ originatore & $€ 27.493 .481$ & $€ 5.546 .418$ \\
$\begin{array}{l}\text { Risparmio annuo } \\
\text { Risparmio cumulato }\end{array}$ & $€ 5.200 .228$ & $€ 11.565 .058$ \\
\hline
\end{tabular}

TABELLA VII - Drug Budget Impact: risultati per l'artrite psoriasica

\begin{tabular}{lcc}
\hline Parametri & I anno & V anno \\
\hline $\begin{array}{l}\text { Pazienti totali in trattamento } \\
\text { con adalimumab }\end{array}$ & $\mathbf{4 . 1 9 2}$ & $\mathbf{4 . 1 9 2}$ \\
$\begin{array}{l}\text { Pazienti trattati con adalimumab } \\
\text { biosimilare }\end{array}$ & 1.331 & 3.551 \\
$\begin{array}{l}\text { Pazienti trattati con adalimumab } \\
\text { originatore }\end{array}$ & 2.860 & 641 \\
$\begin{array}{l}\text { Scenario senza biosimilare } \\
\text { (solo originatore) }\end{array}$ & $€ 52.695 .407$ & $€ 52.695 .407$ \\
$\begin{array}{l}\text { Scenario con biosimilare } \\
\text { (originatore + biosimilare) }\end{array}$ & $€ 47.841 .987$ & $€ 39.750 .922$ \\
- di cui costo pazienti trattati & & \\
$\quad$ con biosimilare & $€ 11.882 .030$ & $€ 31.690 .388$ \\
- di cui costo pazienti trattati & & \\
$\quad$ con originatore & $€ 35.959 .957$ & $€ 8.060 .534$ \\
$\begin{array}{l}\text { Risparmio annuo } \\
\text { Risparmio cumulato }\end{array}$ & $€ 4.853 .420$ & $€ 12.944 .484$ \\
\hline
\end{tabular}

all'originatore. Nel corso dei 5 anni il risparmio del SSN dovuto al graduale aumento di utilizzo del biosimilare salirebbe da €31.859.059 del primo anno a €74.083.472 del quinto anno, con un risparmio cumulato nel quinquennio di $€ 260.776 .329$. Nel quinto anno di osservazione il risparmio complessivo annuo per ogni paziente trattato con adalimumab sarebbe di circa €3.185 (= €74.083.472 / 23.250 pazienti).

In tutte le Tabelle da $\mathrm{V}$ a $\mathrm{X}$, per consentire una rappresentazione leggibile nell'ambito di una pagina di testo, sono riportati solo l'anno iniziale e finale del quinquennio considerato nel modello. La patologia con il maggior numero di pazienti trattati con adalimumab è l'artrite reumatoide con 9.920 casi (Tab. V), seguita dalla malattia di Crohn con 4.385 casi (Tab. IX),
TABELLA VIII - Drug Budget Impact: risultati per la psoriasi

\begin{tabular}{lcc}
\hline Parametri & I anno & V anno \\
\hline $\begin{array}{l}\text { Pazienti totali in trattamento con } \\
\text { adalimumab }\end{array}$ & $\mathbf{6 6 9}$ & $\mathbf{6 6 9}$ \\
$\begin{array}{l}\text { Pazienti trattati con adalimumab } \\
\text { biosimilare }\end{array}$ & 276 & 605 \\
$\begin{array}{l}\text { Pazienti trattati con adalimumab } \\
\text { originatore }\end{array}$ & 393 & 64 \\
$\begin{array}{l}\text { Scenario senza biosimilare } \\
\text { (solo originatore) }\end{array}$ & $€ 8.405 .330$ & $€ 8.405 .330$ \\
$\begin{array}{l}\text { Scenario con biosimilare } \\
\text { (originatore + biosimilare) }\end{array}$ & $€ 7.399 .169$ & $€ 6.201 .104$ \\
$\begin{array}{l}\text { - di cui costo pazienti trattati con } \\
\text { biosimilare }\end{array}$ & $€ 2.463 .259$ & $€ 5.396 .334$ \\
$\begin{array}{l}\text { - di cui costo pazienti trattati con } \\
\text { originatore }\end{array}$ & $€ 4.935 .910$ & $€ 804.770$ \\
$\begin{array}{l}\text { Risparmio annuo } \\
\text { Risparmio cumulato }\end{array}$ & $€ 1.006 .160$ & $€ 2.204 .225$ \\
\hline
\end{tabular}

dall'artrite psoriasica con 4.192 casi (Tab. VII) e dalla spondilite anchilosante con 3.613 (Tab. VI). Psoriasi e colite ulcerosa evidenziano le casistiche più basse con 669 (Tab. VIII) e 472 (Tab. X) casi, rispettivamente. Come fatto per l'analisi complessiva, ipotizzando che nei 5 anni di osservazione si realizzino i tassi di uptake riportati in Tabella II, nel quinto e ultimo anno sarà mediamente passato al biosimilare l'87,4\% (range: $83,2-97,6 \%$ ) dei pazienti in trattamento con adalimumab.

Come precedentemente riportato, il costo di trattamento complessivo delle sei patologie con adalimumab, nell'ipotesi di impiegare esclusivamente l'originatore, ammonterebbe a €292.285.991 (Tab. IV). A questo riguardo, le patologie a maggior costo sarebbero l'artrite reumatoide ( $€ 124.706 .884$, Tab. V), la malattia di Crohn (€55.124.927, Tab. IX) e l'artrite psoriasica (€52.695.407, Tab. VII). Grazie al graduale aumento dei consumi del biosimilare di adalimumab, il risparmio cumulato nei 5 anni per l'artrite reumatoide sarebbe pari a $€ 96.958 .781$, seguito dalla malattia di Crohn con €64.370.585, dall'artrite psoriasica con $€ 43.280 .643$, dalla spondilite anchilosante con €41.476.489, dalla psoriasi con €7.924.827 e dalla colite ulcerosa con $€ 6.765 .005$ (Tab. da V a X).

\section{Discussione}

La presente analisi è stata condotta con lo scopo di valutare l'impatto economico per il SSN successivo all'autorizzazione del biosimilare di adalimumab, ipotizzandone l'espansione sul mercato farmaceutico nazionale rispetto a pazienti sia che iniziano la prima linea di trattamento biologico con adalimumab (naïve population) sia già in trattamento con biologico (switch population).

I tassi epidemiologici, i tassi annui di uptake del biosimilare rispetto all'originatore di adalimumab, insieme al minore prezzo del biosimilare, mostrano, complessivamente, nel quinquennio un risparmio cumulato a vantaggio del SSN di circa 261 milioni di euro; tale risparmio passa dal 10,9\% (€31.859.059) del primo anno al 25,3\% (€74.083.472) del quinto anno. 
TABELLA IX - Drug Budget Impact: risultati per la malattia di Crohn

\begin{tabular}{lcc} 
Parametri & I anno & V anno \\
\hline $\begin{array}{l}\text { Pazienti totali in trattamento } \\
\text { con adalimumab }\end{array}$ & $\mathbf{4 . 3 8 5}$ & $\mathbf{4 . 3 8 5}$ \\
$\begin{array}{l}\text { Pazienti trattati con adalimumab } \\
\text { biosimilare }\end{array}$ & 2.647 & 4.279 \\
$\begin{array}{l}\text { Pazienti trattati con adalimumab } \\
\text { originatore }\end{array}$ & 1.738 & 106 \\
$\begin{array}{l}\text { Scenario senza biosimilare } \\
\text { (solo originatore) }\end{array}$ & $€ 55.124 .927$ & $€ 55.124 .927$ \\
$\begin{array}{l}\text { Scenario con biosimilare } \\
\text { (originatore + biosimilare) }\end{array}$ & $€ 45.475 .107$ & $€ 39.523 .455$ \\
$\begin{array}{l}\text { - di cui costo pazienti trattaticon } \\
\text { biosimilare }\end{array}$ & $€ 23.624 .465$ & $€ 38.195 .164$ \\
- di cui costo pazienti trattaticon & & \\
$\quad$ originatore & $€ 21.850 .642$ & $€ 1.328 .291$ \\
$\begin{array}{l}\text { Risparmio annuo } \\
\text { Risparmio cumulato }\end{array}$ & $€ 9.649 .819$ & $€ 15.601 .472$ \\
\hline
\end{tabular}

TABELLA X - Drug Budget Impact: risultati per la colite ulcerosa

\begin{tabular}{lcc}
\hline Parametri & I anno & V anno \\
\hline $\begin{array}{l}\text { Pazienti totali in trattamento } \\
\text { con adalimumab }\end{array}$ & $\mathbf{4 7 2}$ & $\mathbf{4 7 2}$ \\
$\begin{array}{l}\text { Pazienti trattati con adalimumab } \\
\text { biosimilare }\end{array}$ & 273 & 457 \\
$\begin{array}{l}\text { Pazienti trattati con adalimumab } \\
\text { originatore }\end{array}$ & 198 & 14 \\
$\begin{array}{l}\text { Scenario senza biosimilare } \\
\text { (solo originatore) }\end{array}$ & $€ 5.928 .658$ & $€ 5.928 .658$ \\
$\begin{array}{l}\text { Scenario con biosimilare } \\
\text { (originatore + biosimilare) }\end{array}$ & $€ 4.931 .604$ & $€ 4.260 .923$ \\
$\begin{array}{l}\text { - di cui costo pazienti trattaticon } \\
\text { biosimilare }\end{array}$ & $€ 2.440 .965$ & $€ 4.082 .912$ \\
$\begin{array}{l}\text { - di cui costo pazienti trattaticon } \\
\text { originatore }\end{array}$ & $€ 2.490 .640$ & $€ 178.011$ \\
$\begin{array}{l}\text { Risparmio annuo } \\
\text { Risparmio cumulato }\end{array}$ & $€ 997.054$ & $€ 1.667 .736$ \\
\hline
\end{tabular}

Tra le sei indicazioni terapeutiche, a fronte del maggior numero potenziale di pazienti in trattamento con il biosimilare, l'artrite reumatoide determina nel quinquennio il maggiore risparmio cumulato per il SSN con circa 97 milioni di euro, seguita dalla malattia di Crohn con circa 64 milioni di euro.

Ad oggi, non è possibile confrontare questi risultati con quelli di altre analisi condotte in ambito nazionale, se non indirettamente in termini di potenziale espansione del mercato biosimilare e dei risparmi associati. I risultati qui presentati sembrerebbero quantomeno ricalcare il trend di risparmio evidenziato dall'analisi finanziaria condotta da Marcellusi et al. dove, lungo un orizzonte temporale di sei anni, è stato valutato l'impatto economico aggregato dei biosimilari di infliximab ed etanercept nel trattamento dell'artrite reumatoide, della psoriasi, delle malattie infiammatorie intestinali e dell'artrite psoriasica (16). In virtù della disponibilità dei biosimilari di infliximab e di etanercept, concludono gli autori, si potrebbero ottenere risparmi cumulativi compresi tra i 132 e i 296 milioni di euro (16).

Poiché costruito con lo scopo di stimare l'impatto economico sulla spesa farmaceutica pubblica derivante dall'utilizzo del biosimilare rispetto all'originatore di adalimumab, nel presente Drug Budget Impact sono state semplificate alcune variabili di contesto di seguito brevemente richiamate.

I tassi di uptake del biosimilare e le quote di mercato di adalimumab originatore, seppur stimate da un panel di esperti e riscontrate con gli attuali dati di mercato, non hanno tenuto conto di eventuali specifiche politiche regionali quali determinanti di mercato/spesa. Infatti, Regioni contraddistinte da politiche a favore di alti tassi di uptake del biosimilare ne massimizzerebbero l'impatto economico. Ciononostante, le stime delle quote di mercato di adalimumab e dei tassi di uptake del biosimilare fornite dal panel di esperti hanno cercato, per quanto possibile, di riprodurre differenti comportamenti prescrittivi (per singola indicazione) adottabili nella pratica clinica.

Le quote di mercato di adalimumab originatore sul totale dei pazienti trattati con biologici sono state assunte costanti nel quinquennio di osservazione, senza considerare eventuali variazioni di mix tra l'originatore e altre molecole per la stessa indicazione. Questa impostazione, lungo i 5 anni di osservazione, ha di fatto escluso un'eventuale riduzione del numero di soggetti in trattamento con adalimumab dovuta alla commercializzazione/utilizzo di molecole alternative. II modello ha inoltre escluso la possibilità che l'introduzione di adalimumab biosimilare possa comportare il passaggio ad adalimumab di alcuni pazienti in trattamento coi DMARD ( $D i$ sease-Modifying Anti-Rheumatic Drugs), in virtù della sensibile diminuzione di prezzo del biosimilare. L'inclusione di tali variazioni modificherebbe verosimilmente l'impatto economico determinato dal passaggio al biosimilare di adalimumab qui presentato.

La switch population è stata suddivisa in due sottogruppi di pazienti. II primo costituito da soggetti in trattamento continuativo con adalimumab, per i quali il passaggio al biosimilare può avvenire solo tramite switch; il secondo dai pazienti che hanno fallito una prima linea biologica per i quali il passaggio al biosimilare (di adalimumab) può avvenire fin da subito al posto dell'originatore (switch win). Per questo secondo sottogruppo, nella pratica clinica, differentemente da quanto ipotizzato nel $\mathrm{DBI}$, potrebbe accadere che, a seguito del fallimento di una prima linea biologica con un anti TNF- $\alpha$, la scelta di una seconda linea ricada su un farmaco con un differente meccanismo d'azione. Anche questo aspetto potrebbe determinare un impatto sugli attuali risultati.

II DBI, inoltre, non ha considerato quali variabili del prezzo di adalimumab (biosimilare e originatore) né la presenza di gare regionali, né l'effetto competitivo che il prezzo del biosimilare potrebbe esercitare sulle politiche di prezzo adottabili da parte dell'azienda titolare dell'immissione in commercio dell'originatore. Ciò è stato fatto nell'ottica di presentare una panoramica nazionale e generale dell'impatto finanziario del biosimilare di adalimumab, prescindendo dalla presenza di differenti politiche di prezzo adottate in ambito regionale o 
di differenti strategie di prezzo adottate in ambito aziendale, entrambe attualmente poco prevedibili.

\section{Conclusioni}

Nel complesso, ovvero per la maggior parte delle ipotesi, si può affermare che l'impostazione conservativa del modello utilizzato possa avere stimato un risultato inferiore a quello che si potrebbe verificare nella pratica clinica. Lo studio ha comunque evidenziato gli apprezzabili risultati positivi, in termini di risparmio della spesa farmaceutica pubblica, attesi dall'immissione di adalimumab biosimilare sul mercato. Più in generale, è quindi realistico prevedere che i biosimilari forniranno un sempre più importante contributo al controllo della spesa farmaceutica pubblica.

\section{Disclosures}

Financial support: This research was commissioned by Sandoz S.p.A.

Conflict of interest: None of the authors has financial interest related to this study to disclose.

\section{Bibliografia}

1. De Vol R, Bedroussian A. An unhealthy America: The economic burden of chronic disease. The Milken Institute, 2007. Disponibile sul sito: http://assets1c.milkeninstitute.org/assets/ Publication/ResearchReport/PDF/chronic_disease_report.pdf Accesso: Marzo 2019.

2. Uhlig T, Loge JH, Kristiansen IS et al. Quantification of reduced health-related quality of life in patients with rheumatoid arthritis compared to the general population. J Rheumatol. 2007;34(6):1241-7.

3. Lundkvist J, Kastang F, Kobelt G. The burden of rheumatoid arthritis and access to treatment: health burden and costs. Eur J Health Econ. 2008;8 Suppl 2:549-60.

4. Marcellusi R, Viti R, Capone A et al. Costi diretti e indiretti assorbiti dalle patologie HCV-indotte in Italia: stima basata su una metodologia probabilistica di Cost of Illness. PharmacoEcon Ital Res Artic. 2014; online, DOI: 10.1007/s40276-014-0023-9

5. Allegri M, Lucioni C, Mazzi S et al. Il costo sociale del dolore cronico in Italia. Glob Reg Health Technol Assess. 2014; online, DOI: $10.5301 /$ GRHTA.5000187
6. Kobelt G, Berg J, Lindgren $P$ et al. Costs and quality of life in multiple sclerosis in Europe: method of assessment and analysis. Eur J Health Econ. 2006;7:S5-S13.

7. de Waure C, Sferrazza A, Gualano MR et al. Epidemiologia e burden of disease dell'artrite reumatoide. Ital J Public Health. 2010;7(2) Suppl 2:S3-S13.

8. Chen ML, Shah V, Patnaik $R$ et al. Bioavailability and bioequivalence: an FDA regulatory overview. Pharm Res. 2001;18:1645-50.

9. European Medicine Agency. Committee for Medicinal Products for Human Use (CHMP). Guidelines on Similar Biological Medicinal Products. CHMP/437/04, London, 2005. Disponibile sul sito: http://www.ema.europa.eu/docs/en_GB/document_ library/Scientific_guideline/2009/09/WC500003517.pdf Accesso: Marzo 2019.

10. European Medicine Agency. Guidelines on similar biological medicinal products, 2014. Disponibile sul sito: http://www. ema.europa.eu/docs/en_GB/document_library/Scientific_guideline/2013/05/WC500142978.pdf Accesso Marzo 2019.

11. European Medicine Agency. Guideline on similar biological medicinal products containing biotechnology-derived proteins as active substance: non-clinical and clinical issues, 2013. Disponibile sul sito: http://www.ema.europa.eu/docs/en_GB/ document_library/Scientific_guideline/2013/06/WC500144124. pdf Accesso Marzo 2019.

12. Farmaci biologici e biosimilari. Scenari terapeutici e stima del risparmio per il Sistema Sanitario italiano. Centro Studi IQVIA Italia. Disponibile sul sito: http://magazine.imshealth. it/report/(IQVIA)_Farmaci_biologici_e_biosimilari.pdf Accesso Marzo 2019.

13. IBG - Italian Biosimilars Group (Assogenerici). Report sul mercato italiano dei farmaci biosimilari nel terzo trimestre 2018. Disponibile sul sito: http://www.italianbiosimilarsgroup. it/en/studi-ed-analisi/biosimilari-in-italia/mercato-italiano-iiitrimestre-2018.html Accesso Marzo 2019.

14. Mauskopf JA, Sullivan SD, Annemans L et al. Principles of good practice for budget impact analysis: report of the ISPOR Task Force on Good Research Practices-budget impact analysis. Value Health. 2007;10(5):336-47.

15. Sullivan SD, Mauskopf JA, Augustovski F et al. Budget impact analysis principles of good practice: report of the ISPOR 2012 Budget Impact Analysis Good Practice II Task Force. Value Health. 2014;17(1):5-14.

16. Marcellusi A, Bianchi L, Gasbarrini A et al. Impatto economico dei biosimilari in Italia: passato, presente e futuro di infliximab ed etanercept. Glob Reg Health Technol Assess. 2017;4(1):e175-e186. 